\title{
Optimising environmental risk assessments
}

\author{
Accounting for ecosystem services helps to translate broad policy protection goals into specific \\ operational ones for environmental risk assessments
}

\author{
Yann Devos ${ }^{1}$, Jörg Romeis ${ }^{2}$, Robert Luttik ${ }^{3}$, Angelo Maggiore ${ }^{4}$, Joe N Perry ${ }^{5}$, Reinhilde Schoonjans ${ }^{4}$, \\ Franz Streissl ${ }^{6}$, José V Tarazona ${ }^{6}$ \& Theo CM Brock ${ }^{7}$
}

$\mathrm{R}$ egulated products such as genetically modified organisms (GMOs), plant protection products (PPPs) or feed additives for livestock are subject to an environmental risk assessment before they can be approved for use in agriculture. This assessment aims to evaluate any possible risk that the deployment of such products may pose to the environment. Robust environmental risk assessments require an explicit formulation of potential problems to identify plausible and relevant exposure scenarios and potential adverse effects from predicted exposures. The actual risk is then characterised by testing specific hypotheses about the likelihood and severity of these adverse effects [1-4]. The ultimate decision on what is an acceptable level of risk, and thus whether a GMO, PPP or feed additive can be commercialised, is taken by risk managers-policymakers and regulatorswho have to weigh different policy options to accept, minimise or reduce the risks that were characterised through the environmental risk assessment.

The first step of an environmental risk assessment is to establish the context for the assessment by identifying which components of the environment-species, habitats, services, etc.-are valued by civil society and/or protected by relevant laws or policies. This exercise establishes the so-called environmental policy protection goals: environmental components that should be protected and taken into account when conducting environmental risk assessments to support regulatory decision-making. These protection goals can vary between jurisdictions, but their overall aim is to minimise harm to the environment, including biodiversity and ecosystems, caused by human activities.

However, policy protection goals, such as protecting biodiversity, are often too generic and vague to be useful for scientific risk assessment, and need to be translated into specific, operational ones. Because protecting everything, everywhere, forever is not always tenable, operational protection goals, also termed specific protection goals, have to delineate the environmental components that need to be protected, where and over what time period, and the maximum impacts that can be tolerated $[1,2]$. The definition of operational protection goals is therefore crucial for environmental risk assessments and regulatory decision-making [4]. Since 2010, the European Food Safety Authority (EFSA), which provides scientific advice to European policymakers on possible risks to human, plant and animal health and the environment from the deployment of GMOs, PPPs and feed additives, has been developing a science-based framework to specify operational protection goals in its environmental risk assessments (http://www. efsa.europa.eu/sites/default/files/corporate_ publications/files/583e.pdf). This framework accounts for the importance of ecosystems and biodiversity, and focuses in particular on the benefits these provide to humans, the so-called ecosystem services.
$\mathrm{T}$ he Millennium Ecosystem Assessment, which is widely applied, distinguishes four categories of ecosystem services: provisioning services (products such as food/feed, water, fibre or energy); regulating services, such as pollination, pest control, water and air purification; cultural services (recreation, tourism or cultural heritage); and supporting services that are necessary for the other ecosystem services to function, such as nutrient cycling, soil formation, oxygen production or habitat provision. Irrespective of the various classifications for ecosystem services (Box 1), ecosystem services are highly interconnected and interdependent, and involve multiple species, ecosystems, environmental compartments in ecosystems, and habitats. Different types of ecosystems will therefore offer different types of ecosystem services, and the role of a specific species in providing a specific ecosystem service may differ between ecosystems [3].

“

“...policy protection goals, such as protecting biodiversity, are often too generic and vague to be useful for scientific risk assessment..."

Although biodiversity is usually not explicitly mentioned as an ecosystem service, it is the source of many ecosystem

\footnotetext{
1 European Food Safety Authority (EFSA), Genetically Modified Organisms Unit, Parma, Italy. E-mail: yann.devos@efsa.europa.eu

2 Agroscope, Institute for Sustainability Sciences (ISS), Zurich, Switzerland

3 Almere, The Netherlands

4 European Food Safety Authority (EFSA), Scientific Committee and Emerging Risks Unit, Parma, Italy

5 Oaklands Barn, Broome, Norfolk, UK

6 European Food Safety Authority (EFSA), Pesticides Unit, Parma, Italy

7 Wageningen, The Netherlands

DOI 10.15252/embr.201540874 | Published online 11 August 2015
} 


\section{Box 1. Examples of classifications for ecosystem services}

- The Millennium Ecosystem Assessment (MEA; http://www.millenniumassessment.org/en/index html) distinguishes four categories of ecosystem services, which are widely applied, comprising provisioning services (products obtained from ecosystems including food/feed, water, fibre and energy), regulating services (benefits obtained from the regulation of ecosystem processes; for example, pollination, control of pests and diseases, purification of water and air), cultural services (non-material benefits obtained from ecosystems through recreation and aesthetic experiences; they include ecotourism, cultural heritage, knowledge systems, and spiritual and religious values) and supporting services (services necessary for the production of all other ecosystem services such as nutrient cycling, soil formation, production of atmospheric oxygen and habitat provision).

- The Economics of Ecosystems and Biodiversity (TEEB; http://www.teebweb.org/) approach updates the MEA classification through the explicit recognition of habitat services, which are not part of the MEA concept, as an additional ecosystem service category.

- The Common International Classification of Ecosystem Services (CICES; http://cices.eu/) builds on the MEA and TEEB classifications: it merges the four MEA categories into three categories: provisioning services; regulating and maintenance services; and cultural services. The supporting services as proposed by the MEA are treated as part of the underlying structures, processes and functions that characterise ecosystems, and are pooled with the regulating services to tailor the classification to economic accounting.

- Other classifications offer more economically focussed definitions and distinguish between intermediate services, final services, goods/benefits and well-being value. Final ecosystem services are those components of nature that are directly enjoyed, consumed or used to enhance human well-being, while others are referred to as intermediate services. Goods are all-use and non-use, material and non-material outputs from ecosystems that have a value for people. Values may be economic, health or shared/social [3].

services and plays an essential role in ecosystems and their ability to provide benefits to humans $[5,6]$.

The ecosystem service concept has gained wide acceptance within the international scientific community, and is currently widely applied by policymakers to protect biodiversity and safeguard the sustainability of ecosystems (http://ec.europa.eu/environment/integration/research/newsalert/pdf/ ecosystem_services_biodiversity_IR11_en.pdf). However, putting the ecosystem service concept into practice can be challenging owing to the above-mentioned complexity of various ecosystem components and their interactions, and the limited understanding of how the deployment of regulated products may impact ecosystem services across different spatial scales [3].

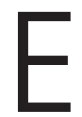

FSA's ecosystem service-based framework to define operational protection goals follows three sequential steps: identifying relevant ecosystem services potentially impacted by the use of regulated products; identifying service-providing units - structural and functional components of biodiversity-that provide or support these ecosystem services; and specifying the level of protection for these serviceproviding units. Here, we use the honeybee (Apis mellifera L.) as an example of a service-providing unit to illustrate how the proposed framework would work in practice. Honeybee colonies worldwide have declined substantially during the past years, a phenomenon that has been attributed to exposure to multiple-biological, chemical, nutritional and environmental-stressors [7]. There is growing concern that further decline could have severe ecological and economic impacts, as honeybees are important pollinators. Some of the drivers that might be contributing to the decline in honeybee populations are regulated products within EFSA's remit. Consequently, environmental risk assessments need to formulate operational goals so as to protect honeybees and safeguard the sustainability of pollination services.

The first step of EFSA's framework thus identifies all relevant ecosystem services that may be adversely affected by the regulated product. In agro-ecological landscapes, these services comprise the provision of food/feed; biological control of pests, weeds and diseases; pollination of crops; nutrient regulation; and cultural values. With regard to honeybees, pollination is a highly relevant ecosystem service in arable landscapes, since many type of plants including commercially important fruit and vegetable crops depend on pollination for seed set and fruit production. Loss of pollination services can therefore have severe negative impacts that could significantly affect the maintenance of wild plant diversity, wider ecosystem stability, crop production, food security and human welfare [7]. In addition, honeybees are also valued for another ecosystem service: the provision of hive products such as honey and beeswax.

The second step selects service-providing units on a case-by-case basis that support the relevant ecosystem services that have been identified and are potentially impacted by the regulated product. In the case of pollination, this involves several animal taxa, of which bees-managed, native and wild bees-are the most important ones. Bees are obligate florivores throughout their life cycle, with both adults and larvae depending on pollen and nectar. The honeybee is also the most important commercially managed pollinator [8,9]. In addition, honeybees contribute to diversity indirectly by pollinating and propagating many plant species, which serve as a food source for other pollinators. Thus, A. mellifera is a key service-providing unit in agro-ecosystems, though it cannot be considered representative of all bee taxa, because populations of wild bees usually comprise different functional and life-history traits. It is therefore necessary to take other service-providing units into consideration when ensuring the protection of pollination services and biodiversity.

The third step of EFSA's framework specifies the degree and parameters of protection for each service-providing unit based on five dimensions. This step necessitates dialogue with risk managers, because it involves normative considerations, which cannot be accounted for by risk assessors alone $[2,10]$. The first dimension is the ecological entity of the serviceproviding unit and represents the level of biological organisation-individuals, populations, functional groups and communitiesthat necessitates protection; in most cases, this is populations. Most ecosystems have functional redundancy if several species are able to perform the same function. Since these species are at least partly substitutable, it is unlikely that a temporal decrease in one population will have biologically relevant effects on the ecosystem service. However, some species contribute to an ecosystem service in unique ways and their loss from a community would cause detectable changes. Moreover, if species are legally protected or have specific needs, the ecological entity needs to 
be at the individual level. Following the example of honeybees, EFSA defined the ecological entity as the colony and its forager population for the provision of pollination services and hive products $[8,9]$.

cc

"... putting the ecosystem service concept into practice can be challenging owing to the above-mentioned complexity of various ecosystem components and their interactions..."

The second dimension is the attribute of the service-providing unit to protect, and refers to the parameter of the selected ecological entity that requires protection: survival, abundance or reproduction. In the case of honeybees, it includes survival and development of colonies. Additional attributes are abundance/biomass and reproduction owing to their importance for the development and long-term survival of colonies $[8,9]$.

The third dimension, the maximum tolerable impact, defines the degree of change of the selected attribute that is regarded tolerable in terms of biological relevance. For honeybees, the viability of each colony, the pollination services provided by it and its yield in terms of hive products all depend on the colony's strength and, in particular, on the number of individuals. Based on the available information, EFSA considered that impacts of less than $7 \%$ on colony size compared with non-exposed colonies as having negligible adverse effects on ecosystem services and the long-term survival of the colony. Impacts greater than $35 \%$ would most likely affect ecosystem services and long-term colony sustainability.

The two final dimensions are the spatial and temporal scale of protection. The spatial scale specifies the area of the maximum tolerable impact: within a field, a field edge or a region for example. In the case of honeybees, the spatial scale was defined as colonies that occur at the edge of agricultural fields and are exposed to the regulated product $[8,9]$. The temporal scale defines the duration of the maximum tolerable effect. For honeybees, EFSA set the temporal scale as a few days for small impacts [8,9].

Following a dialogue with risk managers, EFSA indicated that the in-field exposure of honeybees to a regulated product should not exceed a level that could lead to impacts greater than $7 \%$ in $90 \%$ of the colonies at the edge of exposed agricultural fields $[8,9]$.

$\mathrm{T}$ he above-mentioned dimensions are tightly interrelated, and the choice made for one specific dimension directly influences others. For example, a maximum impact that can be tolerated over a short term may not be acceptable if it continues over a longer time. Small effects on population density could be acceptable at a local scale for a medium period of time, as long as it does not affect a population on a regional scale. It is therefore important that the relationship between the various dimensions is presented to risk managers in a concise and transparent manner. Moreover, the rationale to justify specific choices for each dimension should be made explicitly, using relevant criteria whenever possible (Table 1). Based on life-history traits and the potential for recovery, for instance, it can be justified to require a higher level of protection for an important species within a service-providing unit that has a long lifecycle and few offspring and which is restricted to a small geographical area, compared to a globally distributed, mobile species that has many offspring and a short recovery time. Some species within a service-providing unit may be more vulnerable than others owing to a higher likelihood of exposure to the regulated product in the occupied habitat; higher sensitivity to the regulated product; higher susceptibility to stressors (including habitat loss); or lower potential for recovery.

cc

....EFSA's ecosystem

service-based framework offers

a practical approach that can

be used to define operational protection goals ..."

For example, solitary bees, which predominantly forage in agricultural crops and/or field margins, are more vulnerable to stress than honeybees. Honeybees form colonies, which provide an exceptionally resilient buffer against losses of foragers and workers. Honeybee colony losses are compensated for through the production of many workers daily, the physiological and behavioural plasticity in honeybees (i.e., young workers can start foraging earlier, old workers can return to hive duties), and

Table 1. Examples of criteria to justify made choices for each of the five dimensions specifying operational protection goals (as proposed by the European Food Safety Authority, EFSA).

\begin{tabular}{|c|c|c|}
\hline Dimension & Choice & Criteria to justify choices \\
\hline $\begin{array}{l}\text { Ecological } \\
\text { entity to protect }\end{array}$ & $\begin{array}{l}\text { Individual; (meta)population; } \\
\text { community; functional group; ecosystem }\end{array}$ & $\begin{array}{l}\text { Cultural value (e.g. conservation species, aesthetic species); potential for recovery; } \\
\text { functional redundancy; legal considerations }\end{array}$ \\
\hline $\begin{array}{l}\text { Attribute to } \\
\text { protect }\end{array}$ & $\begin{array}{l}\text { Survival; growth; reproduction; abundance; } \\
\text { biomass; biodiversity; foraging behaviour }\end{array}$ & $\begin{array}{l}\text { Life-history traits of service-providing unit; chemical-physical properties of environment; } \\
\text { ethical and legal considerations }\end{array}$ \\
\hline $\begin{array}{l}\text { Maximum } \\
\text { tolerable impact }\end{array}$ & Negligible; small; medium; large & $\begin{array}{l}\text { Life-history traits of service-providing unit; potential for recovery; level of endangerment } \\
\text { and ecological relevance of the subpopulation; landscape structures; ecological } \\
\text { characteristics of the environment }\end{array}$ \\
\hline $\begin{array}{l}\text { Spatial scale } \\
\text { of protection }\end{array}$ & $\begin{array}{l}\text { Crop/field; edge of field/field margin; nearby } \\
\text { off-crop; farm/holding/production unit; } \\
\text { watershed; landscape; region; continent }\end{array}$ & $\begin{array}{l}\text { Mobility and dispersal ability of relevant life stages of service-providing unit; habitat } \\
\text { and landscape characteristics, in particular its fragmentation }\end{array}$ \\
\hline $\begin{array}{l}\text { Temporal scale } \\
\text { of protection }\end{array}$ & $\begin{array}{l}\text { Days; weeks; months; seasons; years; } \\
\text { generations; crop rotations }\end{array}$ & $\begin{array}{l}\text { Temporal scale during which service-providing unit operates; reproduction strategy of } \\
\text { service-providing unit in terms of time and number of offspring; potential for and time } \\
\text { needed for recovery; habitat }\end{array}$ \\
\hline
\end{tabular}


honey and pollen stores in beehives to survive the winter. In contrast, solitary bees have no such buffering capacity and are likely to be more vulnerable to the same level of stress than colony-forming bees (http:// www.easac.eu/fileadmin/Reports/Easac_15 ES_web_complete_01.pdf). Vulnerable species within a service-providing unit therefore require a higher level of protection. The use of clear criteria to specify the five dimensions will facilitate communication and discussion between risk assessors and risk managers, and help to clarify the rationale for the choices being made [10].

l n conclusion, EFSA's ecosystem servicebased framework offers a practical approach that can be used to define operational protection goals in a systematic, comprehensive and transparent manner for different regulated products. It requires the identification of relevant ecosystem services and service-providing units that may be harmed, and the definition of the level of protection based on five dimensions. The framework therefore helps to identify and account for trade-offs between ecosystem services, such as between food production and conservation of biodiversity. Such tradeoffs may lead to conflicts of interest; hence, stakeholder consultation and communication can contribute to informed and transparent choices on which ecosystem services to protect and prioritise.

\section{"If what constitutes harm is not defined at the beginning of the environmental risk assessment, then one cannot discover harmful effects by scientific research"}

The proposed framework also provides an easy-to-understand tool and a common language, which should facilitate the communication between stakeholders: citizens, academia, risk managers, risk assessment bodies, industry and non-governmental organisations. Improved communication will help to clarify divergent positions on what stakeholders value and why. This communication is also essential to reach agreement on operational protection goals, which must be set before environmental risk assessments are conducted, as they define the framework in which risk assessors operate. If what constitutes harm is not defined at the beginning of the environmental risk assessment, then one cannot discover harmful effects by scientific research $[2,10]$. Thus, reaching agreement on operational protection goals and the criteria to use to define them will increase the value of environmental risk assessments by providing information necessary for effective regulatory decision-making. The information gathered during environmental risk assessment and its interpretation are then used to evaluate the likelihood and severity of adverse effects on what is desirable to protect.

\section{Disclaimer}

The views expressed in this publication are those from the authors and do not necessarily represent the official position of EFSA. EFSA assumes no responsibility or liability for any errors or inaccuracies that may appear.

\section{Acknowledgements}

We thank the experts of the Protection Goals Working Group of EFSA's Scientific Committee for inspiring discussions that helped to develop this publication.

\section{Conflict of interest}

The authors declare that they have no conflict of interest.

\section{References}

1. Nienstedt KM, Brock TCM, van Wensum J, Montforts M, Hart A, Aagaard A, Alix A,
Boesten J, Bopp SK, Brown C et al (2012) Development of a framework based on an ecosystem services approach for deriving specific protection goals for environmental risk assessment of pesticides. Sci Total Environ 415: 31-38

2. Sanvido O, Romeis J, Gathmann A, Gielkens M, Raybould A, Bigler F (2012) Evaluating environmental risks of genetically modified crops - ecological harm criteria for regulatory decision-making. Environ Sci Pol 15: $82-91$

3. Maltby L (2013) Ecosystem services and the protection, restoration, and management of ecosystems exposed to chemical stressors. Environ Toxicol Chem 32: 974-983

4. Garcia-Alonso M, Raybould A (2014) Protection goals in environmental risk assessment: a practical approach. Transgenic Res 23 : 945-956

5. Cardinale BJ, Duffy JE, Gonzalez A, Hooper DU, Perrings C, Venail P, Narwani A, Mace GM, Tilman D, Wardle DA et al (2012) Biodiversity loss and its impact on humanity. Nature 486: 59-67

6. Mace GM, Norris K, Fitter AH (2012) Biodiversity and ecosystem services: a multilayered relationship. Trends Ecol Euol 27: 19-26

7. Potts SG, Biesmeijer JC, Kremen C, Neumann P, Schweiger O, Kunin WE (2010) Global pollinator declines: trends, impacts and drivers. Trends Ecol Euol 25: 345-353

8. EFSA (European Food Safety Authority) (2012) Scientific Opinion on the science behind the development of a risk assessment of Plant Protection Products on bees (Apis mellifera, Bombus spp. and solitary bees). EFSA J 10: $1-275$

9. EFSA (European Food Safety Authority) (2013) EFSA Guidance Document on the risk assessment of plant protection products on bees (Apis mellifera, Bombus spp. and solitary bees). EFSA J 11: 1-268

10. Devos Y, Sanvido O, Tait J, Raybould A (2014) Towards a more open debate about values in decision-making on agricultural biotechnology. Transgenic Res 23: 933-943 